\title{
Buckling of a Holey Column
}

DOI:

10.1039/C6SM00948D

\section{Document Version}

Accepted author manuscript

Link to publication record in Manchester Research Explorer

\section{Citation for published version (APA):}

Pihler-Puzovic, D., Hazel, A., \& Mullin, T. (2016). Buckling of a Holey Column. Soft Matter, 12, 7112-7118. https://doi.org/10.1039/C6SM00948D

\section{Published in:}

Soft Matter

\section{Citing this paper}

Please note that where the full-text provided on Manchester Research Explorer is the Author Accepted Manuscript or Proof version this may differ from the final Published version. If citing, it is advised that you check and use the publisher's definitive version.

\section{General rights}

Copyright and moral rights for the publications made accessible in the Research Explorer are retained by the authors and/or other copyright owners and it is a condition of accessing publications that users recognise and abide by the legal requirements associated with these rights.

\section{Takedown policy}

If you believe that this document breaches copyright please refer to the University of Manchester's Takedown Procedures [http://man.ac.uk/04Y6Bo] or contact uml.scholarlycommunications@manchester.ac.uk providing relevant details, so we can investigate your claim.

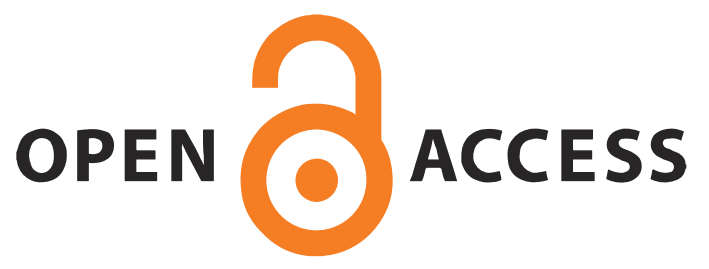




\title{
Journal Name
}

\section{ARTICLE TYPE}

Cite this: DOl: 10.1039/ $\times x \times x \times x \times x \times x \quad$ Buckling of a Holey Column ${ }^{\dagger}$

\author{
D. Pihler-Puzović, ${ }^{* a}$ A. L. Hazel, ${ }^{b}$ and T. Mullin ${ }^{a}$
}

Received Date

Accepted Date

DOI: $10.1039 / x x x x x x x x x x$

www.rsc.org/journalname

\begin{abstract}
We report the results from a combined experimental and numerical investigation of buckling in a novel variant of an elastic column under axial load. We find that including a regular line of centred holes in the column can prevent conventional, global, lateral buckling. Instead, the local microstructure introduced by the holes allows the column to buckle in an entirely different, internal, mode in which the holes are compressed in alternate directions, but the column maintains the lateral reflection symmetry about its centreline. The internal buckling mode can be accommodated within a smaller external space than the global one; and it is the preferred buckling mode over an intermediate range of column lengths for sufficiently large holes. For very short or sufficiently long columns a modification of the classical, global, lateral buckling is dominant.
\end{abstract}

\section{Introduction}

Buckling instabilities are found in a wide range of problems in soft matter such as the loading of rods ${ }^{1}$, fibres ${ }^{2}$ and chains ${ }^{3}$ embedded in soft matrices. They serve as prototypical models of root structures, micro-tubules and muscles respectively. Buckling of elastic structures is a well developed field in engineering 4 with applications in large scale structures such as aircraft, bridges and skyscrapers but similar approaches have been equally successful in the engineering of DNA ${ }^{5}$ for example. Early investigations were primarily concerned with the prevention of buckling, but much recent work has focused on harnessing the effect ${ }^{6}$ with novel applications in soft robotics ${ }^{7}$, and nature inspired photonics $^{8-10}$. Lattice structures formed by perforated elastomers have attracted a great deal of recent attention ${ }^{11-15}$, in part because despite being geometrically simple they exhibit non-trivial buckling modes. Moreover, the reported effects are length-scale independent, suggesting the same instabilities could control optical solitons ${ }^{16}$ at the microscale as well as potentially diverting damaging earthquake vibrations ${ }^{17}$ over kilometre scales.

In this paper we demonstrate that the introduction of a sim-

\footnotetext{
${ }^{a}$ Manchester Centre for Nonlinear Dynamics and School of Physics and Astronomy, University of Manchester, Oxford Road, Manchester, M13 9PL, United Kingdom. Email: D.Pihler-Puzovic@manchester.ac.uk

${ }^{b}$ Manchester Centre for Nonlinear Dynamics and School of Mathematics, University of Manchester, Oxford Road, Manchester, M13 9PL, United Kingdom

$\dagger$ Electronic Supplementary Information (ESI) available: Videos of quasi-steady buckling with evolution of strain energy. See DOI: 10.1039/b000000x/
}

ple geometric modification to the canonical buckling problem of a thin elastic column under axial compression, the Euler strut ${ }^{18}$, can dramatically change the response to loading. Specifically, the inclusion of a regular line of circular holes induces the buckled states shown in the central image of the numerical results in figure 1 and also in experiments as in figure 2. The well-known laterally-deflected buckled state remains, see the righthand image in figure 1, but a new alternating state appears. In this, the holes transform into an array of mutually orthogonal ellipses and the column remains straight. Physically, the modification introduces an internal structure that can support localised buckling in addition to the global (lateral) buckling. Mathematically, the approximate continuous (vertical translation) symmetry of the unbuckled state in a solid column is replaced by a discrete (permutation) symmetry in the holey column, leading to a new and exquisitely rich solution structure.

Competition between localised and global buckling modes has been found to occur in composites, such as biological tissue, where a thin layer of stiff material is embedded within an elastic host of lower stiffness ${ }^{19}$. In those cases, the competition is between the buckling of the thin layer and the buckling of the entire structure. However, in the present study, the change in effective (bending) stiffness is induced purely through local geometry rather than a change in material properties. 


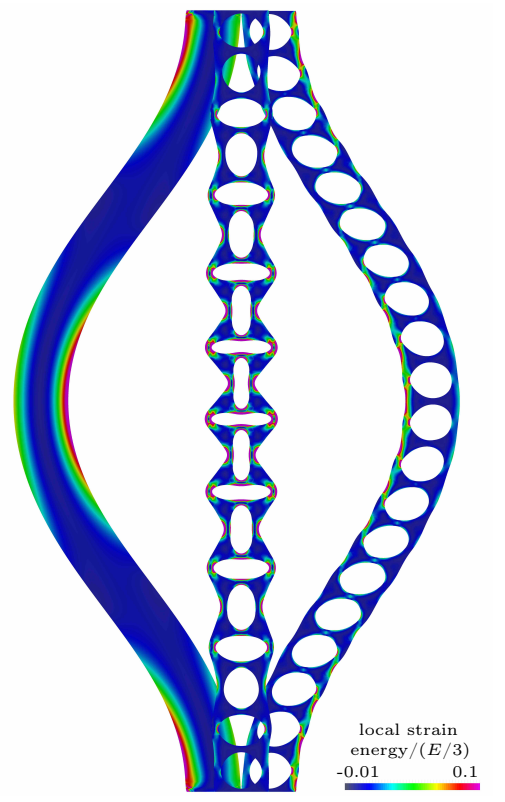

Fig. 1 Centre \& right: two buckled states for a column with 20 regular holes; left: lateral buckling of the corresponding solid column. All states are at the same compression, $\varepsilon=0.093$. The colour map shows the local strain energy normalized by the shear modulus $E / 3$. Left-right symmetry is quantified by $S m=\frac{L-R}{L+R}$, where $L(R)$ is the total strain energy in the region of the column that was located to the left (right) of the centreline in the undeformed configuration. $S m \approx 10^{-10}$ in the symmetric central state; 0.85 in the nonsymmetric rightmost state.

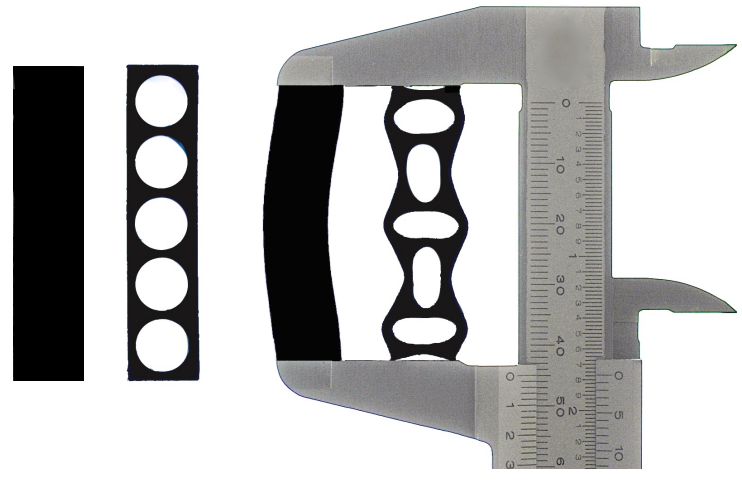

Fig. 2 An illustrative example of the alternating state in a holey column compressed by a vernier caliper. The equivalent solid column is shown alongside and undeformed samples are on the left.

\section{Experimental Methods}

We performed experiments on a set of samples with up to eight holes. Regular samples were moulded using the hyperelastic material extra hard Sid AD Special (Feguramed GmbH). The material was formed by thoroughly mixing quantities of degassed polyvinyl base and siloxane catalyst in a 1:1 volume ratio. The liquid mixture was poured into 3D printed moulds and left to set for 15 minutes. The samples were gently removed from the moulds to avoid tearing and any samples with visible imperfections were discarded. Four different samples were produced for each geometry and measured from calibrated images captured using a Nikon D300s still camera which had a resolution of $0.05 \mathrm{~mm}$ per pixel. An LED light box was used to provide uniform back lighting. The average hole radius $R$, the distance between hole centres $a$ and the sample length $l$, width $w$ and depth $d$ were measured *

Each of the samples was compressed using an Instron 3345 machine with a $5 \mathrm{~N}$ load cell. The force required to achieve a given level of compression was measured to within $0.0025 \mathrm{~N}$. The compression rate was $0.1 \mathrm{~mm} / \mathrm{min}$, but we also performed experiments at a rate of $0.5 \mathrm{~mm} / \mathrm{min}$ and obtained the same results. Thus, we conclude that the loading was sufficiently slow to be considered quasi-static. Samples were placed within a machined perspex channel to facilitate good alignment of the sample and contact surface during compression; and to ensure that the top and bottom surfaces of the samples remained flat, which helped prevent out-of-plane buckling. The out-of-plane mode is not the preferred mode, but can be forced when the alignment is poor. The channel was attached to the Instron machine and lowered onto a perspex indenter. The perspex was stiffer than our samples by factor of $10^{4}$, so its deformation was negligible. We also found that lubricating the top and bottom surfaces of the samples with Vaseline helped to make the forcing uniform.

In the theoretical model we assume a Neo-Hookean, incompressible hyperelastic material ${ }^{20,21}$ under plane strain. Hence, the second Piola-Kirchhoff stress tensor $\sigma^{i j}=(E / 3) g^{i j}-p G^{i j}$, where $p$ is the solid pressure, $E$ is the Young's modulus, $g^{i j}$ and $G^{i j}$ are the contravariant undeformed and deformed metric tensors respectively. The principle of virtual displacements and the incompressibility constraint yield the required governing equa-

\footnotetext{
* Average measurements for the samples with (a) 2 holes are: $R=4.27 \pm 0.08 \mathrm{~mm}$, $a=9.9 \pm 0.05 \mathrm{~mm}, l=20.98 \pm 0.07 \mathrm{~mm}, w=11.14 \pm 0.05 \mathrm{~mm}$ and $d=$ $6.87 \pm 0.29 \mathrm{~mm}$; (b) 3 holes are: $R=4.2 \pm 0.05 \mathrm{~mm}, a=9.83 \pm 0.05 \mathrm{~mm}$, $l=30.83 \pm 0.05 \mathrm{~mm}, w=11.25 \pm 0.05 \mathrm{~mm}$ and $d=7.28 \pm 0.06 \mathrm{~mm}$; (c) 5 holes are: $R=4.23 \pm 0.05 \mathrm{~mm}, a=9.85 \pm 0.05 \mathrm{~mm}, l=50.64 \pm 0.05 \mathrm{~mm}, w=$ $11.12 \pm 0.05 \mathrm{~mm}$ and $d=7.12 \pm 0.11 \mathrm{~mm}$; (d) 8 holes are: $R=4.19 \pm 0.05 \mathrm{~mm}$, $a=9.83 \pm 0.05 \mathrm{~mm}, l=80.24 \pm 0.15 \mathrm{~mm}, w=11.25 \pm 0.05 \mathrm{~mm}$ and $d=7.12 \pm 0.05 \mathrm{~mm}$. The average sample size, also used in the theoretical model, is: $R=4.21 \pm 0.03 \mathrm{~mm}, a=9.84 \pm 0.02 \mathrm{~mm}, w=11.19 \pm 0.07 \mathrm{~mm}$, $d=7.1 \pm 0.17 \mathrm{~mm}$ and $l-N \times a=1.38 \pm 0.18 \mathrm{~mm}$.
} 
tions:

$$
\int \sigma^{i j} \delta \epsilon_{i j} \mathrm{~d} V_{0}=0 \text { and } \int(\operatorname{det} G-1) \delta p \mathrm{~d} V_{0}=0,
$$

where $\epsilon_{i j}$ is the Green-Lagrange strain tensor, $\operatorname{det} G$ is the $\operatorname{de}$ terminant of the deformed metric tensor, $V_{0}$ is the undeformed volume and the Einstein summation convention is used. The $\mathrm{C}++$ library oomph- $l i \mathrm{~b}^{22}$ was used to solve the variational form above via a finite-element approximation in two-dimensions with quadratic interpolation for the displacements and linear interpolation for the pressure. Experimental observations and the good agreement between the theory and experimental data, see figure 3 , indicates that the third, out-of-plane, dimension plays no significant role, as also found in related buckling problems ${ }^{12,15,23}$.

We used the average experimental geometry in the model and each sample comprised $N$ identical elemental cells, with hole radius, $R=4.21 \mathrm{~mm}$, distance between the hole centres, $a=9.84 \mathrm{~mm}$, and sample width, $w=11.19 \mathrm{~mm}$. The results for samples with three or fewer holes were sensitive to the precise details of the geometry at the constrained boundaries and an extra $0.69 \mathrm{~mm}$ of elastic material was added at each end of the model sample to model the experimental samples more closely. A structured mesh of quadrilateral elements was used to discretise each elemental cell and this discretisation was repeated throughout the domain to ensure that the appropriate symmetries were present. The side boundaries of the domain were traction-free; the bottom boundary was pinned to ensure zero displacement in the vertical direction and fixed at the left-hand edge; and the compression was induced by prescribing the displacement of the upper boundary, which was constrained to remain horizontal and also fixed at the left-hand edge.

\section{Results \& Discussion}

\subsection{Global stress-strain relationships}

Stress-strain curves corresponding to elastic columns containing between two and eight holes under compression are shown in figure 3. Data is shown from both the experiments and model calculations. The engineering strain, $\varepsilon$, is defined to be the compression of a sample relative to its initial length $l$ and the engineering stress, $\sigma$, is the ratio of the applied force to the initial cross-sectional area in contact with the loader $d \times w$.

For $\varepsilon<0.04$, all samples in figure 3 follow a linear stress-strain relationship with a gradient equivalent to an effective Young's modulus of $213.8 \pm 0.5 \mathrm{kPa}$. Buckling occurs through a symmetrybreaking bifurcation, accompanied by a change in gradient, at $\varepsilon_{c r}\left(\sigma_{c r}\right)$. Both $\varepsilon_{c r}$ and the corresponding $\sigma_{c r}$ decrease with increasing sample length. The error bars in figure 3 represent the standard deviation over the different experimental runs with the different samples. They are only significant in the vertical direction because the critical stress is sensitive to precise details of sample geometry and end loading conditions but the critical strain is not. In the theoretical model, we confirmed that the critical strains for buckling were independent of the discretisation, but the critical loads were sensitive to the third significant figure, indicating a similar sensitivity to that found in the experiments.

Despite the simplifications in the geometry, boundary conditions and constitutive behaviour used in the model, the agreement between the experimental and the numerical results in figure 3 is striking. This confirms the robustness of the phenomena and lends support to the idea that it does not arise from material nonlinearities. These results also indicate that the critical strain and mode shapes are not particularly sensitive to slight imperfections that are inevitably present in the experiments.

Typical post-buckled shapes of the samples, obtained experimentally and in the model are shown below the stress-strain curve in figure /3 with strain values chosen to achieve approximately the same level of hole compression. Buckling involves lateral movement of the whole sample when there are just two holes involved. In this case, the buckling mode is dominated by the

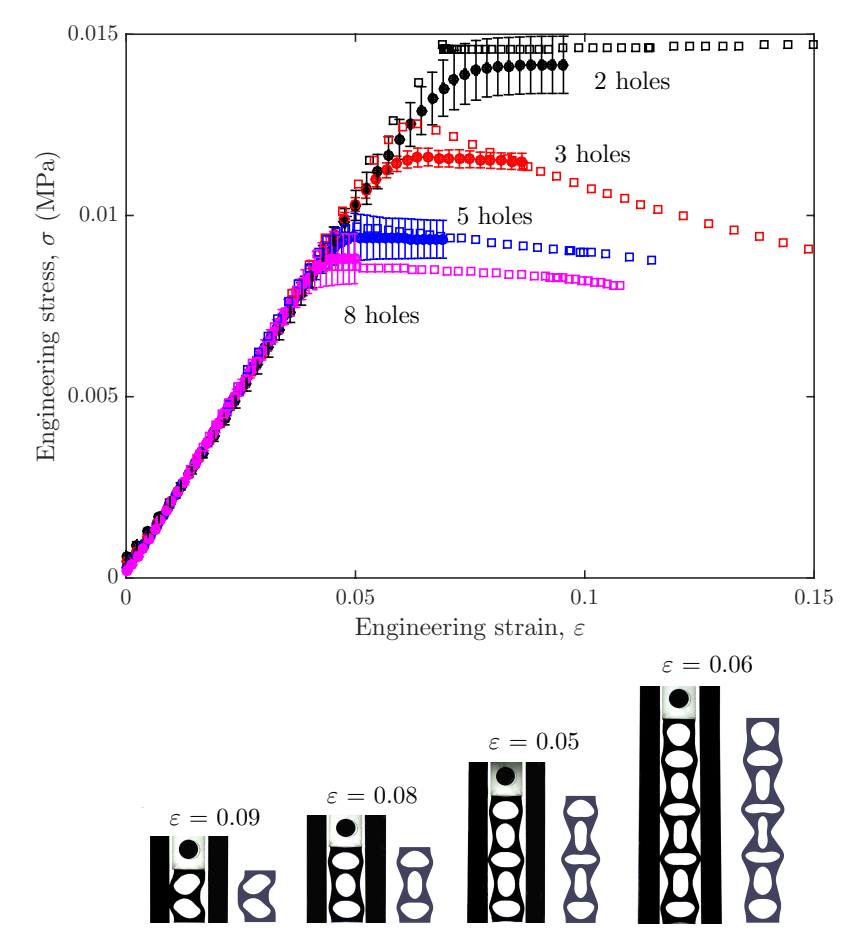

Fig. 3 Stress-strain curves for the compression of the holey columns with 2, 3, 5 and 8 holes, respectively, obtained experimentally (filled markers) and in the theoretical model (empty markers). The errorbars are the standard deviation over 8 experimental runs, 2 for each sample. The transition to the bucked state occurs where the data diverges from the initial linear relationship. Typical buckled states, obtained experimentally (left) and in the theoretical model (right), and the corresponding strain values are shown below. 
boundary conditions. In contrast, the alternating state is the preferred mode for all samples containing between three and eight holes. Indeed, provided the appropriate local critical strain can be induced the alternating buckling should occur for a holey column fabricated from any elastic material. This is because the buckling occurs when it is energetically favourable for regions of the material to bend rather than further compress, i.e. buckling is primarily determined by the local geometry of the sample. By examining the local strain energy, $\sigma^{i j} \epsilon_{i j}$, of a buckled column in the lateral and the alternating states at the same level of compression, it is possible to predict the state that will be observed after buckling. For example, in the case of the sample with six holes, shown in the inset in figure 6 , the total strain energy of the lateral state is greater than that of the alternating state and consequently the holey column buckles in the latter lower-energy state.

We have investigated the role of the material constitutive law by repeating the calculations in figure 3 using an incompressible version of the two-term $I_{1}$-based Rivlin model ${ }^{23}$. In this model $\sigma^{i j}=(E / 3)\left[1+c\left(I_{1}-3\right)\right] g^{i j}-p G^{i j}$, where $I_{1}=g^{i j} G_{i j}$ is a strain invariant and $c$ is a material parameter chosen to be $0.3 / 0.55$ based on experimental data ${ }^{23}$. In compression $\left(I_{1}-3\right)<$ 0 , which means that the additional term leads to an approximate strain softening of the material. In contrast, it is strain hardening under extension. The buckling modes for the different column lengths remain identical and the columns buckle at approximately the same strains, but the critical stresses are slightly increased because sideways extension is more significant than vertical compression globally. The differences between the two models are most pronounced for the two-hole column, which buckles at the largest strains, but even here the critical engineering strain differs by only $\sim 0.001$. We note, however, the the critical strains for buckling are sufficiently small that non-linear effects remain extremely modest.

The results in figure 3 also demonstrate that if the material buckles in the alternating state, then the stress can decrease with subsequent increases in strain. The effect is most pronounced in the theoretical model for the column with three holes. A similar phenomenon is found in the buckling of shells and has been recently observed in the buckling of wide beams and so-called metabeams, in which a more complex internal structure consisting of an array of elliptical holes is introduced ${ }^{15}$. The reduction in stress occurs because the mode accommodates global compression by bending, rather than compressing, the elastic regions around each hole, leading to a large decrease in effective stiffness once the mode becomes accessible to the system. In addition, for an odd number of holes the hole on the horizontal symmetry line of the column plays an important role. If this central hole has buckled with semi-major axis aligned with the external compression, the situation observed experimentally in the case of three holes (see figure 3), then increased buckling due to in- creased force would lead to column extension: a negative effective stiffness. In contrast, when the semi-major axis is normal to the direction of external compression, the situation observed in the case of five holes (see figure 3), the effective stiffness is still reduced, but not as dramatically. The effect can be seen clearly in figure 5 , which presents the complete bifurcation diagram for the perfect (no additional end material) three-hole column.

\subsection{The role of geometry}

As demonstrated in figure 3 , the preferred buckling mode depends on the number of holes in the sample. It also depends on two other geometric parameters: the relative hole size to column width $R / w$, and the relative distance between hole centres to column width $a / w$. For any geometry, as $R / w$ decreases the solid column limit is approached and the classic Euler mode will eventually be preferred. The solid column limit is also approached as $a / w$ increases and the holes move further apart. Thus, we expect the alternating mode to be the preferred mode of buckling for geometries in which the elastic regions between holes are relatively thin and therefore offer limited resistance to bending. The exact values of $R / w$ and $a / w$ at which the preferred buckling modes exchange are not independent and also depend on the number of holes (length) of the sample, see 3.4 for details of the effects of column length on our standard geometry.

\subsection{Bifurcation-theoretical context}

For the solid column, the buckled states correspond to global sinusoidal modes and the strain energy increases with the increasing number of lateral deflections ${ }^{24}$. Thus the mode with a single deflection is always preferred. For the holey column, the buckled states are associated with subgroups of the discrete symmetry group $D_{2} \times S_{N}$, which represents the direct product of the reflection symmetries of the rectangle, $D_{2}$, and all permutations of the $N$ holes, $S_{N}$. Unlike the solid column, where the buckling modes remain the same irrespective of length, in the holey column the number of subgroups increases as $N$ !, leading to increased complexity of possible buckling modes ${ }^{25}$.

We are principally concerned with the first bifurcation that occurs under compression of each column because these give rise to the physically observed (stable) solutions. Once the first bifurcation has occurred all subsequent bifurcations from the trivial branch are of academic interest only because all the solutions are initially unstable. Nonetheless, the forms of the possible solutions can be predicted by using group theory. The general framework for group theoretical analysis of bifurcation phenomena is equivariant bifurcation theory, as described in Golubitsky et al. $(1988)^{25}$. The governing equations of a dynamical system are said to be equivariant under the action of a symmetry group if every solution of the equations is mapped to another solution by the action of every element of the group. The theory can be used 
to predict the symmetries of the eigenfunctions, i. e. those symmetries that can be broken when a single eigenvalue crosses the imaginary axis and therefore occur generically under variation of a single parameter. However, whether or not a particular eigenvalue does actually pass through the origin, leading to bifurcation, can only be determined by solving the specific problem in question.

In the present system, the governing equations are equivariant under the action of the symmetries of the rectangle, $D_{2}$, acting on the entire domain. These are reflections about the horizontal and vertical bisectors and rotation by $180^{\circ}$. In the present problem, there are additional symmetries of the trivial state, however, that give rise to the observed complexity. In the trivial state, each elemental cell has the symmetries of the rectangle $D_{2}$ and the $N$ cells can be freely permuted, assuming that the line between cells remains horizontal. Thus, the symmetry group of the trivial state is $D_{2} \times S_{N}$. This is not a symmetry group of the governing equations, however, because once buckling takes place, the line between elemental cells does not remain horizontal and the cells can no longer be freely permuted. Nonetheless, the eigenfunctions retain the approximate symmetries predicted by theory applied to the group $D_{2} \times S_{N}$.

Standard equivariant bifurcation theory for the global action of $D_{2}$ predicts three possibilities for symmetry-breaking:

- Bifurcation to solutions preserving only the horizontal reflection symmetry,

- Bifurcation to solutions preserving only the vertical reflection symmetry,

- Bifurcation to solutions preserving only the half-turn rotation symmetry.

Lateral buckling preserves the vertical reflection symmetry and always arises through a $D_{2}$-equivariant symmetry-breaking pitchfork bifurcation. In contrast the alternating mode is only $D_{2}$ equivariant when the number of holes is even. In that case, only the horizontal reflection symmetry is preserved and the alternating mode arises through a symmetry-breaking pitchfork bifurcation. For an odd number of holes, the alternating mode is invariant under the symmetry group $D_{2}$ and therefore cannot arise through a symmetry-breaking bifurcation of that group. Instead it represents a loss of the permutation symmetry from the trivial branch. This would be a pitchfork bifurcation if the equations were $D_{2} \times S_{N}$-equivariant. Instead it is actually a transcritical bifurcation, which is the generic form under the additional assumption that the trivial solution always exists. In the experiment both pitchfork and transcritical bifurcations are unfolded by small unavoidable imperfections. Hence it is very difficult to distinguish between the two types of bifurcation in practice.

We now illustrate these theoretical remarks by presenting the bifurcation diagrams that include all bifurcations from the trivial

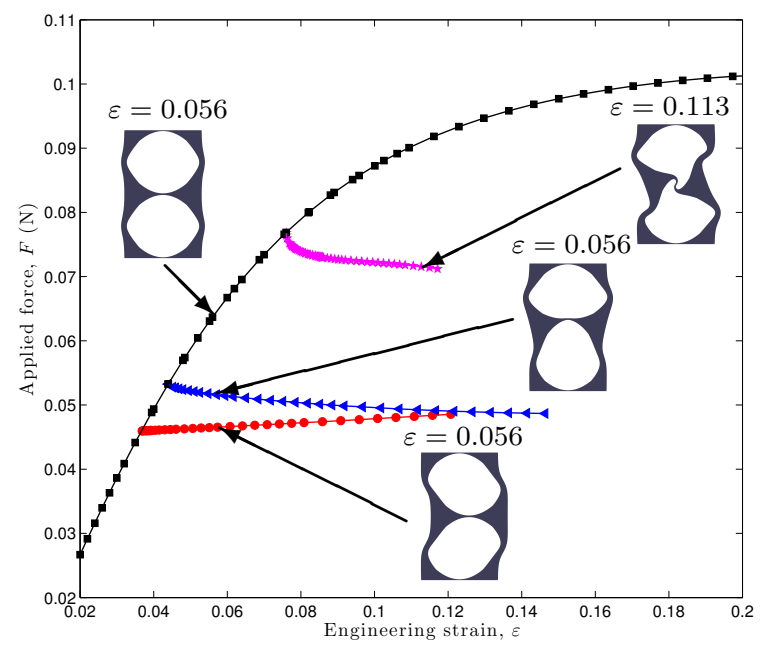

Fig. 4 Computed (theoretical) bifurcation diagram for a perfect column with two holes, each of radius $R=4.8 \mathrm{~mm}$. All bifurcations are pitchforks that break the $D_{2}$ group that acts globally on the entire system. The symmetries of the branches are: trivial (squares), vertical reflection only (circles), horizontal reflection only (triangles) and half-turn rotation only (stars). The lines between discrete points are added to guide the eye. Corresponding solutions are shown near each branch.

branch for "perfect" columns with two and three holes. In both cases eigenfunctions possessing the appropriate symmetries predicted by the equivariant bifurcation theory applied to the full $D_{2} \times S_{N}$ group are found, although the symmetries that are not within $D_{2}$ are only approximate. The number of bifurcations from the trivial branch within the range of physically-allowed parameters (non self-intersecting solutions) increases with increasing hole size, so for illustration of these ideas we present the results for a hole radius of $R=4.8 \mathrm{~mm}$. The distance between hole centres remains $a=9.84 \mathrm{~mm}$ and the sample width remains $w=11.19 \mathrm{~mm}$. The columns are "perfect" in the sense that there is no additional material at the constrained boundaries and the boundary conditions on the upper and lower surfaces are implemented symmetrically.

For two holes, figure 4, there are three bifurcations from the trivial branch, corresponding to the three possibilities from $D_{2}$-equivariant bifurcation theory. In order of increasing compression they are: the lateral mode, the alternating mode and a rotationally-symmetric mode. Eigenfunctions that are $D_{2}$ invariant but break the permutation symmetry of the trivial state are also found but the associated eigenvalues never go unstable.

For three holes, figure 5, there are four bifurcations from the trivial branch. In order of increasing compression these are: a $D_{2}$-equivariant pitchfork bifurcation to the lateral mode; a $D_{2}$ invariant transcritical bifurcation to the alternating mode; a $D_{2}$ equivariant pitchfork bifurcation to a solution that is invariant under rotation; a $D_{2}$-equivariant pitchfork bifurcation that is in- 


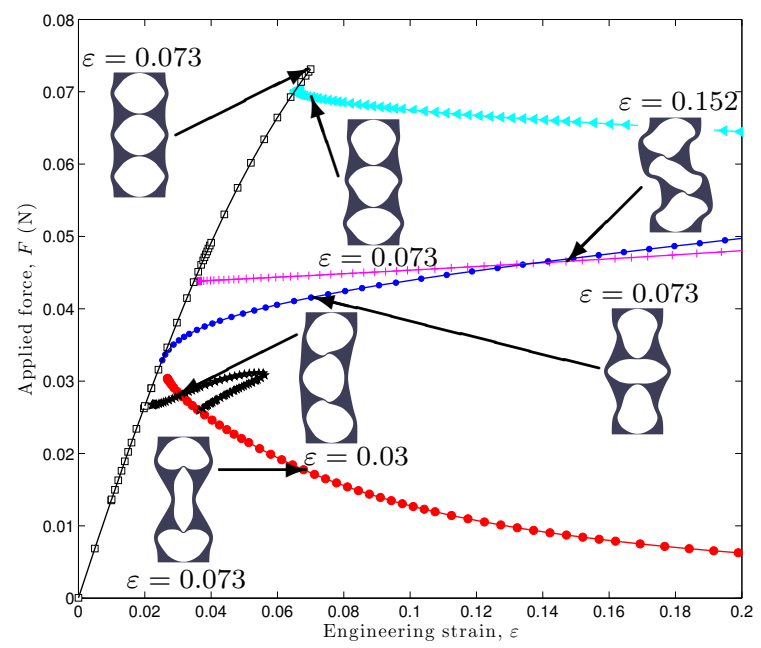

Fig. 5 Computed (theoretical) bifurcation diagram for a perfect column with three holes, each of radius $R=4.8 \mathrm{~mm}$. Three of the bifurcations are pitchforks that break the $D_{2}$ group that acts globally on the entire system. The bifurcation to the alternating mode is actually transcritical with a nearby limit point. This gives rise to two distinct solution branches that are not conjugate under $D_{2}$. Hence, the force required to achieve a given displacement is different for each of the alternating branches. The symmetries of the branches are: trivial (squares), vertical reflection only (stars), half-turn rotation only (crosses), horizontal reflection only (triangles). The alternating mode branches are represented by circles and possess the full $D_{2}$ symmetry, but loss of the approximate permutation symmetry. The lines between discrete points are added to guide the eye. Corresponding solutions are shown near each branch. variant under horizontal reflection and is the perturbed form of the eigenfunction that does not break the $S_{3}$ permutation symmetry. Note that the branch of lateral-mode solutions joins the alternating mode via a secondary bifurcation. Note also that the two branches of alternating mode solutions are not conjugate under the actions of $D_{2}$ and they have quite different forcedisplacement relationships. In contrast, the two branches of the $D_{2}$-equivariant pitchfork bifurcations are indistinguishable in this projection. The different force-displacement relationships for the alternating-mode branches presented in figure 5 are a consequence of the change in orientation of the semi-major axis of the hole on the horizontal symmetry line, as discussed in section 3.1. When the central hole buckles so that its semi-major axis is aligned with the direction of external compression, the lower branch, an increased buckling (increased force) would lead to extension of the column, which results in a negative effective stiffness. When the semi-major axis is normal to the direction of compression, the upper branch, the compression is enhanced, leading to a reduced, but still positive effective stiffness.

\subsection{Predicting the preferred buckling state}

Although this complexity in solution structure is an inherent feature of the holey column, a remarkable observation for the samples in figure 3 is that only the buckling states shown in figure 1 are seen in experiments. These two states represent different extremes: the strain energy is least evenly distributed throughout the material in the lateral state, only the initially vertical side walls of each hole are significantly strained; in contrast, the strain energy is more evenly distributed in the alternating state than any other state - all regions surrounding each hole are under strain. We can predict the preferred buckling state of any column by computing the smaller value of $\varepsilon_{c r}$ corresponding to these two extreme states, keeping the same void to material ratio as in figure 3 but increasing number of holes, see figure 6 .

The data were obtained using the model now in the absence of extra end material to preserve the permutation symmetry and $\varepsilon_{c r}$ was calculated by solving a standard extended system to find points at which the Jacobian (tangent stiffness matrix) has a zero eigenvalue corresponding to the appropriate eigenmode. $\varepsilon_{c r}$ is plotted as a function of the number of holes $N$ on a log-log scale for the lateral and the alternating buckling states. For comparison, we also show $\varepsilon_{c r}$ at the onset of buckling for the corresponding solid columns without holes (in this case $N$ corresponds to the ratio of the sample length to the length of the elemental cell). The theoretical scaling for the lateral state in a solid column with clamped ends gives $\varepsilon_{c r}=4 \pi^{2} I /\left(d w N^{2} a^{2}\right)$, where $I=d w^{3} / 12$ is the area moment of inertia of the cross section of the column ${ }^{24}$. This prediction is demonstrated in figure 6 with the lower dotted line and becomes quantitative once $N \geq 6$.

The critical strain for the lateral state in the holey column also 
exhibits a $1 / N^{2}$ scaling, suggesting that the same essential mechanism is at play as in the solid column. Examination of the strain energy in figures 1 and 6 and the supplementary video material indicates that the deformation is confined to thin columns whose width is of the order of the narrowest cross-section of elastic material. The prediction of the Euler theory in which the area moment of inertia and column width are replaced by those corresponding to the narrowest cross-section, $I_{\min }=\left(w^{3}-8 R^{3}\right) / 12$, $w_{\min }=(w-2 R)$, is shown as the upper dotted line in figure 6 . The numerical data is in excellent accord with the asymptotic prediction, albeit approaching it for much larger $N$ than in the case of the solid column.

The critical strain associated with the lateral state decreases as the length of the column increases, ultimately tending to zero. On the other hand, the critical strain for the alternating buckling state appears to saturate at a constant value as the column length increases. The critical buckling strain for an "infinite" column in which a two-hole sample is subject to periodic boundary conditions in the vertical direction but with traction-free sides is given by $\varepsilon_{c r}^{p}=0.035$, which gives a lower bound for $\varepsilon_{c r}$. Although we might expect $\varepsilon_{c r} \rightarrow \varepsilon_{c p}^{p}$ as $N$ increases, it is not clear that the influence of the boundaries can ever be totally neglected. For the periodic calculations, we used an unstructured mesh of tri-

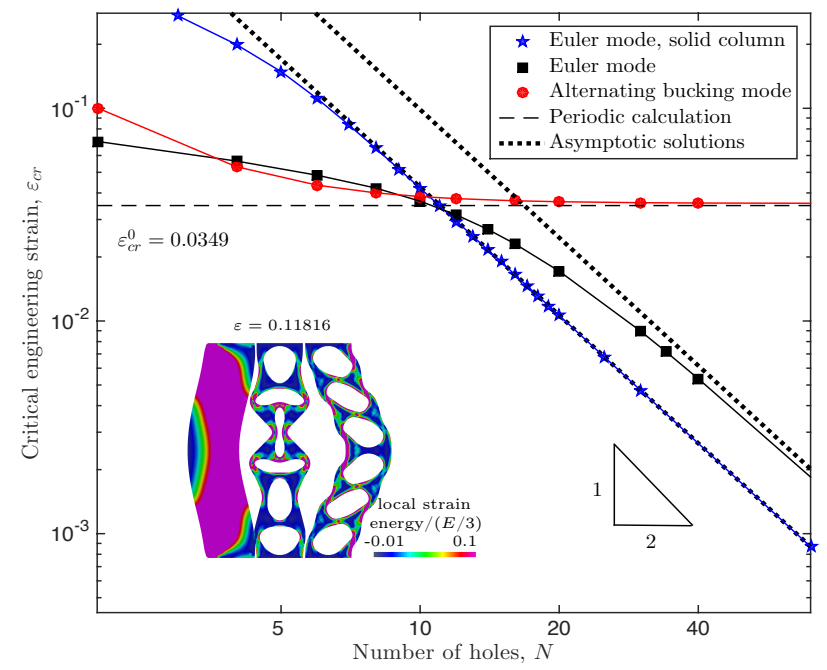

Fig. 6 Log-log plot of $\varepsilon_{c r}$ as a function of $N$ obtained numerically for $(\boldsymbol{\square})$ the lateral and $(\bullet)$ the alternating states in the holey column with the same void to bulk ratio as in figure 3 and $(\star)$ the lateral state in the corresponding solid column. The lines between discrete points are added to guide the eye. The horizontal dashed line, $\varepsilon_{c r}^{p}=0.035$, is $\varepsilon_{c r}$ for the two-hole sample with periodic boundary conditions. The dotted lines are predictions of the Euler theory for columns with fixed ends. The insets are the same states as in figure 1 with 6 holes at $\varepsilon=0.11816$. angles in our numerics and modified the boundary conditions so that material points on the upper and lower boundaries that were originally aligned vertically remained separated by a constant vertical distance. This distance was then decreased to simulate the compression, while the pressures at the upper and lower boundaries were constrained to be the same. The different behaviours for the lateral and alternating states in figure 6 as the column length increases suggest that a sufficiently long holey column will always buckle as an Euler column, unless specifically forced into the alternating buckling state.

\section{Conclusions}

We have shown that a simple modification of the canonical example of mechanical buckling and symmetry-breaking bifurcation, the introduction of a line of holes in a solid column, has non-intuitive and potentially far-reaching consequences. The buckling still occurs via a symmetry-breaking bifurcation, but broken symmetry is not necessarily the left-right reflection symmetry. As a consequence, it is possible to realise a buckled state which has the qualitative form of bamboo. Interestingly the alternating state was previously unreported in related studies of cellular structures with smaller void to material ratios ${ }^{26,27}$. Relatively large void to material ratios are required for the new state to be realised because it involves local bending of the elastic regions between the holes, which is preferred when these elastic regions are thin. This new state can accommodate the same mechanical load within a much more confined space than conventional columns. Localised buckling is observed in soft shell structures and composites, but not in solid columns. One interpretation of our geometric modification is that it introduces shell-like behaviour by adding a second, smaller, lengthscale to the structure. Broadening our approach to include the effects of void fraction, hole shape and hole size distribution will further enhance the diversity of the phenomena potentially allowing custom tuning of buckling states to specific applications. The phenomena also has implications for the widely-used Euler-Greenhill formula ${ }^{28}$ often used in the prediction of the mechanical stability of trees ${ }^{29}$. Our new results suggest that the height at which an elastic column topples could be increased by modifying the internal structure.

\section{Acknowlegements}

The authors thank A. Juel, M. Heil, J. Montaldi, L. Ducloue and C. Johnson, P. Reis for useful discussions and M. Walker and D. Chorlton for technical support.

\section{References}

1 T. Su, J. Liu, D. Terwagne, P. M. Reis and K. Bertoldi, Soft Matter, 2014, 10, 6294-6302. 
2 Y. Zhao, J. Li, Y. Cao and X.-Q. Feng, Soft Matter, 2016, 12, 2086-2094.

3 S. Huang, G. Pessot, P. Cremer, R. Weeber, C. Holm, J. Nowak, S. Odenbach, A. Menzelb and G. Auernhammer, Soft Matter, 2016, 12, 228-237.

4 L. J. Gibson and M. F. Ashby, Cellular Solids, Structure and Properties, Cambridge University Press, Cambridge, 1997.

5 K. Krieger, Nature, 2012, 488, 146-147.

6 J. Shim, S. Shan, A. Košmrlj, S. H. Kang, E. R. Chen, J. C. Weaver and K. Bertoldi, Soft Matter, 2013, 9, 8198-8202.

7 D. Rus and M. T. Tolley, Nature, 2015, 521, 467-475.

8 P. Vukusic and J. R. Sambles, Nature, 2003, 424, 852-855.

9 J. Li, J. Shim, J. Overvelde, J. Deng, X. Zhu, K. Bertoldi and S. Yang, Soft Matter, 2012, 8, 10322.

10 X. Zhu, G. Wu, R. Dong, C. Chen and S. Yang, Soft Matter, 2012, 5, 2412-2418.

11 T. Mullin, S. Deschanel, K. Bertoldi and M. C. Boyce, Phys. Rev. Lett., 2007, 99, 084301.

12 K. Bertoldi, P. M. Reis, S. Willshaw and T. Mullin, Adv. Mater. 2010, 22, 361-366.

13 S. Willshaw and T. Mullin, Soft Matter, 2012, 8, 1747-1750.

14 B. Florijn, C. Coulais and M. van Hecke, Phys. Rev. Lett., 2014, 113, 175503.

15 C. Coulais, J. T. B. Overvelde, L. A. Lubbers, K. Bertoldi and M. van Hecke, Phys. Rev. Lett., 2015, 115, 044301.

16 Y. Kivshar, Adv. Nat. Sci: Nanosci. Nanotechnol., 2014, 5,
013001.

17 S. Brûlé, E. H. Javelaud, S. Enoch and S. Guenneau, Phys. Rev. Lett., 2014, 112, 133901.

18 A. E. H. Love, A Treatise on the Mathematical Theory of Elasisity, Dover Publications, New York, 1944.

19 Y. Li, N. Kaynia, S. Rudykh and M. C. Boyce, Advanced Engineering Materials, 2013, 15, 921-926.

20 R. W. Ogden, Non-linear Elastic Deformations, Dover Publications, New York, 1997.

21 A. E. Green and W. Zerna, Theoretical Elasticity, Oxford University Press, London, 1968.

22 M. Heil and A. L. Hazel, Fluid-Structure Interaction, 2006, pp. 19-49.

23 K. Bertoldi, M. C. Boyce, S. Deschanel, S. M. Prange and T. Mullin, J. Mech. Phys. Solids, 2008, 56, 2642-2668.

24 S. P. Timoshenko and J. M. Gere, Theory of Elastic Stability. Second Edition, McGraw-Hill, New York, 1961.

25 M. Golubitsky, I. N. Stewart and D. G. Schaeffer, Singularities and Groups in Bifurcation Theory, Springer-Verlag, Berlin, 1988.

26 A. M. I. Sweedan, K. M. El-Sawy and M. Ikbal Martini, ThinWalled Struct., 2009, 47, 442-454.

27 A. M. I. Sweedan and K. M. El-Sawy, J. Constr. Steel Res., 2011, 67, 1115-1127.

28 A. G. Greenhill, Proc. Camb. Phil. Soc., 1881, 4, 65-73.

29 K. Niklas, Tree Physiology, 2007, 27, 433-440. 